\title{
Gravitational Energy-Momentum Density in Teleparallel Gravity
}

\author{
V.C. de Andrade, L. C. T. Guillen, and J. G. Pereira \\ Instituto de Física Teórica, Universidade Estadual Paulista, Rua Pamplona 145, 01405-900 São Paulo SP, Brazil
}

(Received 8 December 1999)

In the context of a gauge theory for the translation group, a conserved energy-momentum gauge current for the gravitational field is obtained. It is a true spacetime and gauge tensor, and transforms covariantly under global Lorentz transformations. By rewriting the gauge gravitational field equation in a purely spacetime form, it becomes the teleparallel equivalent of Einstein's equation, and the gauge current reduces to the Møller's canonical energy-momentum density of the gravitational field.

PACS numbers: $04.50 .+\mathrm{h}$

The definition of an energy-momentum density for the gravitational field is one of the oldest and most controversial problems of gravitation. As a true field, it would be natural to expect that gravity should have its own local energy-momentum density. However, it is usually asserted that such a density cannot be locally defined because of the equivalence principle [1]. As a consequence, any attempt to identify an energy-momentum density for the gravitational field leads to complexes that are not true tensors. The first of such attempts was made by Einstein who proposed an expression for the energy-momentum density of the gravitational field which was nothing but the canonical expression obtained from Noether's theorem [2]. Indeed, this quantity is a pseudotensor, an object that depends on the coordinate system. Several other attempts have been made, leading to different expressions for the energy-momentum pseudotensor for the gravitational field [3].

Despite the existence of some controversial points related to the formulation of the equivalence principle [4], it seems true that, in the context of general relativity, no tensorial expression for the gravitational energy-momentum density can exist. However, as our results show, in the gauge context, the existence of an expression for the gravitational energy-momentum density which is a true spacetime and gauge tensor turns out to be possible. Accordingly, the absence of such an expression should be attributed to the general relativity description of gravitation, which seems to not be the appropriate framework to deal with this problem [5].

In spite of some skepticism [1], there has been a continuous interest in this problem [6]. In particular, a quasilocal approach has been proposed recently which is highly clarifying [7]. According to this approach, for each gravitational energy-momentum pseudotensor, there is an associated superpotential which is a Hamiltonian boundary term. The energy momentum defined by such a pseudotensor does not really depend on the local value of the reference frame, but only on the value of the reference frame on the boundary of a region - then its quasilocal character. As the relevant boundary conditions are physically acceptable, this approach validates the pseudotensor approach to the gravitational energy-momentum problem. It should be mentioned that these results were obtained in the context of the general relativity description of gravitation.

In the present work a different approach will be used to reexamine the gravitational energy-momentum problem. Because of the fundamental character of the geometric structure underlying gauge theories, the concept of currents, and, in particular, the concepts of energy and momentum, are much more transparent when considered from the gauge point of view [8]. Accordingly, we are going to consider gravity as described by a gauge theory [9]. Our basic interest will be concentrated on the gauge theories for the translation group [10], and, in particular, on the so-called teleparallel equivalent of general relativity [11]. It is important to remark that this equivalence is true only in the absence of spinor matter fields [12].

Let us start by reviewing the fundamentals of the teleparallel equivalent of general relativity. We use the Greek alphabet $(\mu, \nu, \rho, \ldots=0,1,2,3)$ to denote indices related to spacetime, and the Latin alphabet $(a, b, c, \ldots=0,1,2,3)$ to denote indices related to the tangent space (fiber), assumed to be a Minkowski space with the metric $\eta_{a b}=\operatorname{diag}(+1,-1,-1,-1)$. A gauge transformation is defined as a local translation of the tangent-space coordinates,

$$
\delta x^{a}=\delta \alpha^{b} P_{b} x^{a},
$$

with $P_{a}=\partial / \partial x^{a}$ the translation generators, and $\delta \alpha^{a}$ the corresponding infinitesimal parameters. Denoting the gauge potentials by $A^{a}{ }_{\mu}$, the gauge covariant derivative of a general matter field $\Psi$ is [13]

$$
\mathcal{D}_{\mu} \Psi=h_{\mu}^{a} \partial_{a} \Psi
$$

where

$$
h^{a}{ }_{\mu}=\partial_{\mu} x^{a}+c^{-2} A^{a}{ }_{\mu}
$$

is a nontrivial tetrad field, with $c$ the speed of light. From the covariance of $\mathcal{D}_{\mu} \Psi$, we obtain the transformation of the gauge potentials:

$$
A_{\mu}^{a^{\prime}}=A_{\mu}^{a}-c^{2} \partial_{\mu} \delta \alpha^{a} .
$$


As usual in Abelian gauge theories, the field strength is given by

$$
F_{\mu \nu}^{a}=\partial_{\mu} A_{\nu}^{a}-\partial_{\nu} A_{\mu}^{a},
$$

which satisfies the relation

$$
\left[\mathcal{D}_{\mu}, \mathcal{D}_{\nu}\right] \Psi=c^{-2} F_{\mu \nu}^{a} P_{a} \Psi .
$$

It is important to remark that, whereas the tangent-space indices are raised and lowered with the metric $\eta_{a b}$, the spacetime indices are raised and lowered with the Riemannian metric

$$
g_{\mu \nu}=\eta_{a b} h^{a}{ }_{\mu} h_{\nu}^{b} .
$$

A nontrivial tetrad field induces on spacetime a teleparallel structure which is directly related to the presence of the gravitational field. In fact, given a nontrivial tetrad $h^{a}{ }_{\mu}$, it is possible to define a Cartan connection

$$
\Gamma_{\mu \nu}^{\rho}=h_{a}{ }^{\rho} \partial_{\nu} h_{\mu}^{a},
$$

which is a connection presenting torsion, but no curvature [14]. As a natural consequence of this definition, the Cartan covariant derivative of the tetrad field vanishes identically:

$$
\nabla_{\nu} h_{\mu}^{a} \equiv \partial_{\nu} h_{\mu}^{a}-\Gamma_{\mu \nu}^{\theta} h_{\theta}^{a}=0 .
$$

This is the absolute parallelism condition. The torsion of the Cartan connection is

$$
T_{\mu \nu}^{\rho}=\Gamma_{\nu \mu}^{\rho}-\Gamma_{\mu \nu}^{\rho},
$$

from which we see that the gravitational field strength is nothing but torsion written in the tetrad basis:

$$
F_{\mu \nu}^{a}=c^{2} h^{a}{ }_{\rho} T_{\mu \nu}^{\rho} .
$$

The Cartan connection $\Gamma_{\mu \nu}^{\rho}$ and the Levi-Civita connection of the metric (7), denoted by $\stackrel{\circ}{\Gamma}_{\mu \nu}{ }_{\mu \nu}$, are related by

$$
\Gamma_{\mu \nu}^{\rho}=\stackrel{\circ}{\Gamma}_{\mu \nu}^{\rho}+K_{\mu \nu}^{\rho},
$$

with

$$
K_{\mu \nu}^{\rho}=\frac{1}{2}\left(T_{\mu \nu}^{\rho}{ }_{\nu}+T_{\nu}{ }^{\rho}{ }_{\mu}-T_{\mu \nu}^{\rho}{ }_{\mu \nu}\right),
$$

the contorsion tensor.

The gauge gravitational field Lagrangian is given by [13]

$$
\mathcal{L}_{G}=\frac{h c^{4}}{16 \pi G} S^{\rho \mu \nu} T_{\rho \mu \nu},
$$

where $h=\operatorname{det}\left(h_{\mu}^{a}\right)$, and

$$
S^{\rho \mu \nu}=-S^{\rho \nu \mu} \equiv \frac{1}{2}\left[K^{\mu \nu \rho}-g^{\rho \nu} T_{\theta}^{\theta \mu}+g^{\rho \mu} T_{\theta}^{\theta \nu}\right]
$$

is a tensor written in terms of the Cartan connection only. As usual in gauge theories, it is quadratic in the field strength. By using relation (12), this Lagrangian can be rewritten in terms of the Levi-Civita connection. Up to a total divergence, the result is the Hilbert-Einstein Lagrangian of general relativity

$$
\mathcal{L}=-\frac{c^{4}}{16 \pi G} \sqrt{-g} \stackrel{\circ}{R},
$$

where the identification $h=\sqrt{-g}$ has been made.

By performing variations in relation to the gauge field $A_{a}{ }^{\rho}$, we obtain from the gauge Lagrangian $\mathcal{L}_{G}$ the teleparallel version of the gravitational field equation,

$$
\partial_{\sigma}\left(h S_{a}{ }^{\sigma \rho}\right)-\frac{4 \pi G}{c^{4}}\left(h j_{a}{ }^{\rho}\right)=0,
$$

where $S_{a}{ }^{\sigma \rho} \equiv h_{a}{ }^{\lambda} S_{\lambda}{ }^{\sigma \rho}$. Analogously to the Yang-Mills theories [15],

$$
h j_{a}{ }^{\rho} \equiv-\frac{\partial \mathcal{L}_{G}}{\partial h^{a}{ }_{\rho}}=\frac{c^{4}}{4 \pi G} h h_{a}{ }^{\lambda} S_{\mu}{ }^{\nu \rho} T^{\mu}{ }_{\nu \lambda}-h_{a}{ }^{\rho} \mathcal{L}_{G}
$$

stands for the gravitational gauge current, which in this case represents the energy and momentum of the gravitational field. The term $\left(h S_{a}{ }^{\sigma \rho}\right)$ is called superpotential in the sense that its derivative yields the gauge current $\left(h j_{a}{ }^{\rho}\right)$. Because of the antisymmetry of $S_{a}{ }^{\sigma \rho}$ in the last two indices, $\left(h j_{a}{ }^{\rho}\right)$ is conserved as a consequence of the field equation:

$$
\partial_{\rho}\left(h j_{a}{ }^{\rho}\right)=0 .
$$

Making use of the identity

$$
\partial_{\rho} h \equiv h \Gamma_{\nu \rho}^{\nu}=h\left(\Gamma_{\rho \nu}^{\nu}-K_{\rho \nu}^{\nu}\right),
$$

this conservation law can be rewritten as

$$
D_{\rho} j_{a}{ }^{\rho} \equiv \partial_{\rho} j_{a}{ }^{\rho}+\left(\Gamma_{\lambda \rho}^{\rho}-K_{\lambda \rho}^{\rho}\right) j_{a}{ }^{\lambda}=0,
$$

where $D_{\rho}$ is the teleparallel version of the covariant derivative, which is nothing but the Levi-Civita covariant derivative of general relativity rephrased in terms of the Cartan connection [16]. As can be easily checked, $j_{a}{ }^{\rho}$ transforms covariantly under a general spacetime coordinate transformation, and is invariant under local (gauge) translation of the tangent-space coordinates. This means that $j_{a}{ }^{\rho}$ is a true spacetime and gauge tensor. However, it transforms covariantly only under a global tangent-space Lorentz transformation.

Let us now proceed further and find out the relation between the above gauge approach and general relativity. By using Eq. (8) to express $\partial_{\rho} h_{a}{ }^{\lambda}$, the field equation (16) can be rewritten in a purely spacetime form,

$$
\partial_{\sigma}\left(h S_{\lambda}{ }^{\sigma \rho}\right)-\frac{4 \pi G}{c^{4}}\left(h t_{\lambda}{ }^{\rho}\right)=0,
$$

where now

$$
h t_{\lambda}{ }^{\rho}=\frac{c^{4}}{4 \pi G} h \Gamma_{\nu \lambda}^{\mu} S_{\mu}^{\nu \rho}+\delta_{\lambda}{ }^{\rho} \mathcal{L}_{G}
$$


stands for the teleparallel version of the canonical energymomentum pseudotensor of the gravitational field. Despite not explicitly apparent, as a consequence of the local Lorentz invariance [17] of the gauge Lagrangian $\mathcal{L}_{G}$, the field equation (21) is symmetric in $(\lambda \rho)$. Furthermore, by using Eq. (12), it can be rewritten in terms of the Levi-Civita connection only. As expected, due to the equivalence between the corresponding Lagrangians, it is the same as Einstein's equation:

$$
\frac{h}{2}\left[\stackrel{\circ}{R}_{\mu \nu}-\frac{1}{2} g_{\mu \nu} \stackrel{\circ}{R}\right]=0 .
$$

The canonical energy-momentum pseudotensor $t_{\lambda}{ }^{\rho}$ is not simply the gauge current $j_{a}{ }^{\rho}$ with the algebraic index " $a$ " changed to the spacetime index " $\lambda$." It incorporates also an extra term coming from the derivative term of Eq. (16):

$$
t_{\lambda}{ }^{\rho}=h_{\lambda}^{a} j_{a}{ }^{\rho}+\frac{c^{4}}{4 \pi G} \Gamma_{\lambda \nu}^{\mu} S_{\mu}^{\nu \rho} .
$$

We see thus clearly the origin of the connection term which transforms the gauge current $j_{a}{ }^{\rho}$ into the energymomentum pseudotensor $t_{\lambda}{ }^{\rho}$. Through the same mechanism, it is possible to appropriately exchange further terms between the derivative and the current terms of the field equation (21), giving rise to different definitions for the energy-momentum pseudotensor, each one connected to a different superpotential $\left(h{S_{\lambda}}^{\rho \sigma}\right)$. Like the gauge current $\left(h j_{a}{ }^{\rho}\right)$, the pseudotensor $\left(h t_{\lambda}{ }^{\rho}\right)$ is conserved as a consequence of the field equation:

$$
\partial_{\rho}\left(h t_{\lambda}{ }^{\rho}\right)=0 .
$$

However, in contrast to what occurs with $j_{a}{ }^{\rho}$, due to the pseudotensor character of $t_{\lambda}{ }^{\rho}$, this conservation law can not be rewritten with a covariant derivative.

Because of its simplicity and transparency, the teleparallel approach to gravitation seems to be much more appropriate than general relativity to deal with the energy problem of the gravitational field. In fact, Møller already noticed a long time ago that a satisfactory solution to the problem of the energy distribution in a gravitational field could be obtained in the framework of a tetrad theory. In our notation, his expression for the gravitational energymomentum density is [18]

$$
h t_{\lambda}{ }^{\rho}=\frac{\partial \mathcal{L}}{\partial \partial_{\rho} h^{a}{ }_{\mu}} \partial_{\lambda} h^{a}{ }_{\mu}+\delta_{\lambda}{ }^{\rho} \mathcal{L},
$$

which is nothing but the usual canonical energymomentum density yielded by Noether's theorem. Using for $\mathcal{L}$ the gauge Lagrangian (14), it is an easy task to verify that Møller's expression coincides exactly with the teleparallel energy-momentum density appearing in the field equations (21) and (22). Since $j_{a}{ }^{\rho}$ is a true spacetime tensor, whereas $t_{\lambda}{ }^{\rho}$ is not, we can say that the gauge current $j_{a}{ }^{\rho}$ is an improved version of the Møller's energy-momentum density $t_{\lambda}{ }^{\rho}$. Mathematically, they can be obtained from each other by Eq. (24). It should be remarked, however, that both of them transform covariantly only under global tangent-space Lorentz transformations. This is, we believe, the farthest one can go in the direction of a tensorial definition for the energy and momentum of the gravitational field. The lack of a local Lorentz covariance can be considered as the teleparallel manifestation of the pseudotensor character of the gravitational energy-momentum density in general relativity. Accordingly, we can say that, if it were possible to define a local Lorentz covariant gauge current in the teleparallel gravity, the corresponding general relativity energy-momentum density would be represented by a true spacetime tensor.

The results can be summarized as follows. In the context of a gauge theory for the translation group, we have obtained an energy-momentum gauge current $j_{a}{ }^{\rho}$ for the gravitational field which transforms covariantly under spacetime general coordinate transformations, and is invariant under local (gauge) translations of the tangent-space coordinates. This means essentially that $j_{a}{ }^{\rho}$ is a true spacetime and gauge tensor. By rewriting the gauge field equation in a purely spacetime form, it becomes equivalent to Einstein's equation of general relativity, and the gauge current $j_{a}{ }^{\rho}$ reduces to the canonical energy-momentum pseudotensor of the gravitational field, which coincides with Møller's well-known expression. In the ordinary context of general relativity, therefore, the energy-momentum density for the gravitational field will always be represented by a pseudotensor.

According to the quasilocal approach, to any energymomentum pseudotensor there is an associated superpotential which is a Hamiltonian boundary term [7]. On the other hand, the teleparallel field equations explicitly exhibit both the superpotential and the gravitational energy-momentum complex. We see then that, in fact, by appropriately exchanging terms between the superpotential and the current terms of the field equation (21), it is possible to obtain different gravitational energymomentum pseudotensors with their associated superpotentials. In this context, our results can be rephrased according to the following scheme. First, notice that the left-hand side of the field equation (21) as a whole is a true tensor, though each one of its two terms is not. Then if we extract the spurious part from the first term, so that it becomes a true spacetime and gauge tensor, and add this part to the second term, the energy-momentum density, it becomes also a true spacetime and gauge tensor. We thus arrive at the gauge-type field equation (16), with $\left(h S_{a}{ }^{\sigma \rho}\right)$ as the superpotential, whose corresponding expression for the conserved energy-momentum density for the gravitational field, given by $j_{a}{ }^{\rho}$, though transforming covariantly only under a global tangent-space Lorentz transformation, is a true spacetime and gauge tensor.

The authors would like to thank FAPESP-Brazil, CAPES-Brazil, and CNPq-Brazil for financial support. 
[1] C. W. Misner, K. S. Thorne, and J. A. Wheeler, Gravitation (Freeman, New York, 1973).

[2] See, for example, A. Trautman, in Gravitation: an Introduction to Current Research, edited by L. Witten (Wiley, New York, 1962).

[3] A. Papapetrou, Proc. R. Ir. Acad. A 52, 11 (1948); P. G. Bergmann and R. Thompson, Phys. Rev. 89, 400 (1953); C. Møller, Ann. Phys. (Paris) 4, 347 (1958); L. D. Landau and E. M. Lifshitz, The Classical Theory of Fields (Pergamon Press, Oxford, 1975).

[4] For a critical discussion of the equivalence principle, see the preface of J. L. Synge, Relativity: The General Theory (North-Holland, Amsterdam, 1960).

[5] J. W. Maluf, J. Math. Phys. 36, 4242 (1995).

[6] M. Dubois-Violette and J. Madore, Commun. Math. Phys. 108, 213 (1987); L. B. Szabados, Classical Quantum Gravity 9, 2521 (1992); J. M. Aguirregabiria, A. Chamorro, and K. S. Virbhadra, Gen. Relativ. Gravit. 28, 1393 (1996); T. Shirafuji and G. L. Nashed, Prog. Theor. Phys. 98, 1355 (1997); S. Deser, J. S. Franklin, and D. Seminara, Classical Quantum Gravity 16, 2815 (1999); S. V. Babak and L. P. Grishchuck, gr-qc/9907027.

[7] C. C. Chang and J. M. Nester, Phys. Rev. Lett. 83, 1897 (1999), and references therein.
[8] F. Gronwald and F. W. Hehl, in Proceedings of the 14th School of Cosmology and Gravitation, Erice, Italy, edited by P. G. Bergmann, V. de Sabbata, and H.-J. Treder (World Scientific, Singapore, 1996).

[9] For a general review, see F. W. Hehl, J. D. McCrea, E. W. Mielke, and Y. Ne'emann, Phys. Rep. 258, 1 (1995).

[10] C. Pelegrini and J. Plebanski, Mat. Fys. Skr. Dan. Vidensk. Selsk. 2, No. 4 (1963); K. Hayashi and T. Nakano, Prog. Theor. Phys. 38, 491 (1967); K. Hayashi and T. Shirafuji, Phys. Rev. D 19, 3524 (1979).

[11] J. W. Maluf, J. Math. Phys. 35, 335 (1994).

[12] K. Hayashi, Lett. Nuovo Cimento 5, 529 (1972).

[13] V. C. de Andrade and J. G. Pereira, Phys. Rev D 56, 4689 (1998).

[14] R. Aldrovandi and J. G. Pereira, An Introduction to Geometrical Physics (World Scientific, Singapore, 1995).

[15] See, for example, P. Ramond, Field Theory: A Modern Primer (Addison-Wesley, Redwood, 1989), 2nd ed.

[16] V. C. de Andrade and J. G. Pereira, Int. J. Mod. Phys. D 8, 141 (1999).

[17] See, for example, S. Weinberg, Gravitation and Cosmology (Wiley, New York, 1972), p. 371.

[18] C. Møller, Mat. Fys. Skr. Dan. Vidensk. Selsk. 1, No. 10 (1961). 